\title{
Safety and Plasma Concentrations of a Cyclin-dependent Kinase 9 (CDK9) Inhibitor, FIT039, Administered by a Single Adhesive Skin Patch Applied on Normal Skin and Cutaneous Warts
}

\author{
Eriko Sumi ${ }^{1}$ (D) Takashi Nomura ${ }^{2} \cdot$ Ryuta Asada $^{3} \cdot$ Ryuji Uozumi $^{1} \cdot$ Harue Tada $^{1} \cdot$ Yoko Amino $^{1} \cdot$ Teruo Sawada $^{3}$. \\ Atsushi Yonezawa $^{5} \cdot$ Masatoshi Hagiwara $^{4} \cdot$ Kenji Kabashima ${ }^{2}$
}

Published online: 4 October 2018

(c) The Author(s) 2018

\begin{abstract}
Background Cutaneous warts are caused by human papilloma virus (HPV) infection. FIT039, a specific inhibitor of CDK9, suppresses the proliferation of DNA viruses in vitro.

Purpose We evaluated the safety, plasma concentrations, and efficacy of FIT039 delivered by single application of an adhesive skin patch on normal back skin and cutaneous warts.

Patients and Methods In this placebo-controlled, dose-escalation, open-label, two-cohort phase I/II clinical trial, after a single administration of a 1\% FIT039 patch, 3\% FIT039 patch, or placebo on back skin, patients with cutaneous warts were treated with cryotherapy followed by a $1 \%$ FIT039 patch for $24 \mathrm{~h}$ in the first cohort. In the second cohort, cutaneous warts were treated with cryotherapy followed by a 3\% FIT039 patch for $24 \mathrm{~h}$. Adverse events and adverse drug reactions, the concentrations of FIT039, and surface area of cutaneous warts were evaluated.

Results Neither irritant reactions nor symptoms related to FIT039 occurred when the FIT039 patches were applied to patients' backs or on warts in ten patients. The concentrations of FIT039 were under $0.1 \mathrm{ng} / \mathrm{ml}$ at every time point. The median wart surface area at 1 week after application of the 1\% FIT039 patch was similar to baseline, while that of the $3 \%$ FIT039 patch was smaller than baseline.

Conclusion The FIT039 patch showed no topical or systemic adverse reactions when applied on normal skin or cutaneous warts. The safety and good adherence of the FIT039 patch are encouraging and support further studies to evaluate the efficacy of FIT039 in patients with cutaneous warts.
\end{abstract}

Eriko Sumi and Takashi Nomura contributed equally.

Electronic supplementary material The online version of this article (https://doi.org/10.1007/s40261-018-0712-7) contains supplementary material, which is available to authorized users.

Eriko Sumi

sumieri@kuhp.kyoto-u.ac.jp

1 Institute for Advancement of Clinical and Translational Science (iACT), Kyoto University Hospital, 54

Shogoin-Kawahara-cho, Sakyo-ku, Kyoto 606-8507, Japan

2 Department of Dermatology, Kyoto University Hospital, Kyoto, Japan

3 Innovative and Clinical Research Promotion Center, Gifu University Hospital, Gifu, Japan

4 Anatomy and Developmental Biology, Graduate School of Medicine, Kyoto University Faculty of Medicine, Kyoto, Japan

5 Department of Clinical Pharmacology and Therapeutics, Kyoto University Hospital, Kyoto, Japan

\section{Key Points}

FIT039, a candidate antiviral drug, may be effective against cutaneous warts associated with human papilloma virus (HPV) infection by suppressing HPV replication and hyperproliferation of keratinocytes.

The safety and good adherence of the FIT039 patch applied on normal skin and cutaneous warts that had been pre-treated with cryotherapy shown in the study are encouraging and support further studies. 


\section{Introduction}

Cutaneous warts are a common disease, especially in children and young adults. Warts generally appear on the hands or feet. Cutaneous warts are caused by human papilloma viruses (HPV) infection. HPV types 27, 57, 2, and 1 are the most prevalent types of cutaneous warts in the general European population [1], and HPV types 1, 4, and 65 are more common in Japan [2].

Typically, warts are cleared naturally or after several treatments with cryotherapy using liquid nitrogen or topical salicylic acid. However, the cure rates following cryotherapy and salicylic acid therapy within 3 months in clinical trials vary from 9 to $93 \%$ and from 0 to $87 \%$, respectively [3]. The efficacy of treatments is influenced by the site of the warts, with warts on the sole of the feet (plantar warts) being more resistant to cryotherapy and topical salicylic acid therapy than warts on the hands or other locations [4]. Cryotherapy is associated with pain and is avoided in young children. Moreover, both cryotherapy and topical salicylic acid therapy destroy infected epithelial cells and do not prevent HPV diffusion. One-fourth of patients had one or more new warts during the treatment period in a clinical trial [4]. New therapeutic interventions are required to shorten the treatment period and to prevent the appearance of new warts by suppressing viral replication.

FIT039 is a cyclin-dependent kinase 9 (CDK9) inhibitor and a candidate antiviral drug. CDK9/cyclin T1 (or $\mathrm{T} 2$ ) is a subunit of positive transcription elongation factor $\mathrm{b}(\mathrm{P}-\mathrm{TEFb})$ that initiates the transcriptional elongation of genes [5]. FIT039 inhibits host cell factor CDK9 and suppresses transcription in DNA viruses such as herpes simplex virus, adenovirus, cytomegalovirus, and hepatitis B virus both in vitro and in vivo [6-9].

Though oral administration of FIT039 was effective in an in vivo pharmacological study in a mouse model, we developed FIT039-containing adhesive skin patches that release FIT039 continuously when applied to the skin [9]. Since FIT039 is thought to have an effect not on virus kinase but on human CDK9, topical administration of FIT039 is considered to be rational by exposing infected cells to high concentrations of FIT039. Before the start of this clinical trial, we confirmed that FIT039 is distributed to the stratum corneum, epidermis, and the upper part of the dermis 2-24 h after application of FIT039 ointment on the skin of Sprague-Dawley rats, and that as much as $9 \%$ and $28 \%$ of FIT039 in normal and decornified skin, respectively, was absorbed at $48 \mathrm{~h}$ after the application [9]. So we expect that FIT039 released from the patch applied on the warts reach keratinocytes and epithelial basal cells, and suppress HPV replication and hyperproliferation of keratinocytes regardless of HPV type.
Here, we evaluated the safety, plasma concentrations, and efficacy of FIT039 delivered by a single application of an adhesive skin patch on normal back skin and on cutaneous warts that had been pre-treated with cryotherapy.

\section{Methods}

\subsection{Study Design}

This placebo-controlled, dose-escalation, open-label, twocohort phase I/II clinical trial was conducted at Kyoto University Hospital.

The study was approved by the institutional review board (IRB) at Kyoto University Hospital on 12 February 2016 (K025). The study was conducted in compliance with the study protocol, the Helsinki Declaration and the Ministerial Ordinance on Good Clinical Practice for Drugs (GCP). This study was registered with the UMIN Clinical Trials Registry as UMIN000019866 (http://www.umin.ac.jp/ctr/ index-j.htm).

\subsection{Participants}

Patients aged 20 years or older with cutaneous warts, measuring 4-10 $\mathrm{mm}$ on the major axis and located on the extremities, including the palms and sole of the feet, were recruited. We excluded patients who had an allergy to adhesive skin patches, had severe complication(s), had taken antiviral agents within 4 weeks prior to giving informed consent, and women who could become pregnant.

\subsection{Intervention}

The $1 \%$ and 3\% FIT039 patches contained $72 \mu \mathrm{g}$ and $216 \mu \mathrm{g}$, respectively, of FIT039 in a $1-\mathrm{cm}$ square. Placebo was the same $1-\mathrm{cm}$ square patch as the FIT039 patch but contained no FIT039.

After a single administration of the 1\% FIT039 patch, 3\% FIT039 patch, and placebo on back skin, patients with cutaneous warts were treated with cryotherapy followed by $1 \%$ FIT039 patch application for $24 \mathrm{~h}$ in the first cohort. In the second cohort, cutaneous warts were treated with cryotherapy followed by $3 \%$ FIT039 patch application for $24 \mathrm{~h}$. If patients had another wart more than $5 \mathrm{~cm}$ away from the target wart, a placebo patch was applied to that wart.

\subsection{Outcome Measurements}

The safety endpoint was the incidence of adverse events and adverse drug reactions. Adverse skin reactions were graded according to the criteria of the International Contact 
Dermatitis Research Group (ICRDG) [10]. The efficacy endpoints were surface area and change in the surface area of the treated wart 1 week after application. In addition, plasma FIT039 concentrations were evaluated at 4 and $24 \mathrm{~h}$ after FIT039 application to the back, and at 2, 4, and $24 \mathrm{~h}$ after FIT039 application on cutaneous warts. Blood samples ( $2 \mathrm{ml}$ ) were centrifuged at $3000 \mathrm{rpm}$ for $10 \mathrm{~min}$ at $2-8{ }^{\circ} \mathrm{C}$ to obtain supernatant. The samples were stored at $-65^{\circ} \mathrm{C}$ until FIT039 levels were quantified at Sekisui Medical Co., Ltd. (Tokyo, Japan).

The surface area of a wart in this study was defined by the product of the long and short axes (in millimetres) of the wart. The following formula was used to determine the change in surface area:

Change in surface area $(\%)=[1-($ surface area of the wart at 1 week after application/surface area of the wart at baseline) $] \times 100$.

\subsection{Analysis of Plasma FIT039 Concentrations}

\subsubsection{HPLC-MS/MS Conditions}

A LC-20 AD series unit (Shimadzu Corporation, Kyoto, Japan) with InertSustain C18 column (particle size: $3 \mu \mathrm{m}$, column size: $2.1 \mathrm{~mm} \times 50 \mathrm{~mm}$ : GL sciences, Tokyo, Japan) was employed for liquid chromatography analysis. The autosampler was set at $10{ }^{\circ} \mathrm{C}$, and the column oven was set at $40{ }^{\circ} \mathrm{C}$. The mobile phase was a mixture of water and $1 \%$ formic acid (1000:1, v/v) (A) and acetonitrile (B). The gradient elution program started at a composition of $50 \%$ $\mathrm{B}$ for $0.10 \mathrm{~min}$ (initial condition) and another $1.50 \mathrm{~min}$ and then was ramped to $90 \% \mathrm{~B}$ at $1.50 \mathrm{~min}$ and held for another $2.00 \mathrm{~min}$. The composition of mobile phase $\mathrm{B}$ was maintained at $50 \%$ from $2.01 \mathrm{~min}$ to $3.00 \mathrm{~min}$, and the system was then returned to the initial condition at $3.00 \mathrm{~min}$. An API 4000 triple quadrupole instrument (SCIEX, Framingham, MA, USA) was equipped with an electrospray ionization (ESI) source in the positive mode for mass spectrometric detection. The monitoring ions were FIT039; $\mathrm{m} / \mathrm{z} 316.2$ (Q1) $\rightarrow$ m/z 282.1 (Q3), and IS; $m / z$ 350.1 (Q1) $\rightarrow m / z 239.3$ (Q3). Internal standards (IS) for the analysis were deuterated forms of the analytes (GIF430, prepared in Kyoto University). Analyst software (version 1.6.2) (SCIEX, Framingham, MA, USA) was used for data processing.

\subsubsection{Preparation of Calibration Standard Samples and QC Samples}

Standard stock solution of $100 \mu \mathrm{g} / \mathrm{ml}$ FIT039 and IS stock solution of $100 \mu \mathrm{g} / \mathrm{ml} \mathrm{GIF} 430$ were prepared and stored at $4{ }^{\circ} \mathrm{C} 1$ day before the analysis. Working solutions ranging from 2 to $10,000 \mathrm{ng} / \mathrm{ml}$ for FIT039 and $200 \mathrm{ng} / \mathrm{ml}$ for the
IS were prepared by dissolving FIT039 or GIF430 in acetonitrile. The calibration standards were obtained by diluting FIT039 working solutions with blank plasma to be final concentrations of $0.1,0.2,1,2.5,10,50$, and $100 \mathrm{ng} / \mathrm{ml}$. The quality control (QC) samples at $0.2,2.5$, and $80 \mathrm{ng} / \mathrm{ml}$ of FIT039 were also prepared by the same procedure. All calibration standards and QC samples were prepared on the day of analysis.

\subsubsection{Preparation of Plasma Samples}

Ten microliters of each plasma sample, $20 \mu \mathrm{l}$ of IS working solution, and $90 \mu \mathrm{l}$ of acetonitrile were mixed and centrifuged after being vortexed. The supernatant was pipetted into a polypropylene tube, mixed with $120 \mu \mathrm{l}$ of water, and then used for subsequent analysis.

\subsubsection{Calibration Curve and Accuracy}

The analytical procedure was developed and validated beforehand in terms of selectivity, calibration curve, intraday reproducibility, interday reproducibility, carry over, matrix effect, dilution reproducibility, and stability in accordance with the Guideline on Bioanalytical Method Validation in Pharmaceutical Development issued by PMDA [11].

The calibration curve was calculated by the peak area ratios of FIT039 to IS against the FIT039 concentrations to fit the $[y=a x+b]$ model with a weighting factor of $1 / x^{2}$, where $x$ is the concentration of the analyte. In this analysis, the correlation coefficient $(r)$ should be equal to or more than 0.99 , and accuracy values of more than 6 points including lower and upper limit of quantification should be between $85 \%$ and $115 \%$ for calibration standards. With regard to QC samples, the accuracy values of more than two-thirds of the samples should be between 85 and $115 \%$.

\subsection{Descriptive Analysis}

Analyses of safety and efficacy were based on a June 2017 database lock. The safety analyses included all patients who were enrolled in this study and received trial treatment, and the incidence of adverse events and adverse drug reactions from patients in each cohort was evaluated from baseline to 1 week after patch application on the warts. Plasma FIT039 concentrations at each time point were reported descriptively. We determined the surface area and the change in the surface area of the target warts at baseline and 1 week after application of patch. Efficacy analyses were carried out on all patients who had patch application with adherence to the protocol and summarized using descriptive statistics. All analyses were performed using SAS statistical software, version 9.4 (SAS Institute, Cary, NC, USA). 


\section{Results}

\subsection{Patients}

A total of 11 patients with cutaneous warts were enrolled in the study, with four patients in cohort 1 and seven patients in cohort 2, between 14 July 2016 and 26 April 2017. The flowchart of this study is shown in Fig. 1. In three patients with more than two warts, a placebo patch was applied on the other wart. The FIT039 patch came off of one patient in cohort 1 within $16 \mathrm{~h}$ after application and was included in the safety analysis but not included in the efficacy analysis. A patient in cohort 2 was discontinued before application of the FIT039 patch and was included in neither safety nor efficacy analysis as with previously described criteria. Baseline characteristics of the patients are shown in Table 1.

\subsection{Safety}

No irritant reactions based on ICDRG criteria were seen with the $1 \%$ FIT039 patch, the 3\% FIT039 patch, or placebo after application to back skin. Itching occurred at the sites of the $1 \%$ FIT039 patch and placebo patch in one patient. An event of "erythema only" based on ICDRG criteria was observed when a $1 \%$ FIT039 patch was applied to a wart that was non-serious and unrelated to the trial treatment. Adverse events at the sites of application are summarized in Supplemental Online Resource Table 1a, b. A total of three adverse events, including herpes simplex infection, pain, and contact dermatitis at a site other than the trial patch application site were reported, and all were classed as non-serious and unrelated to the trial treatment.

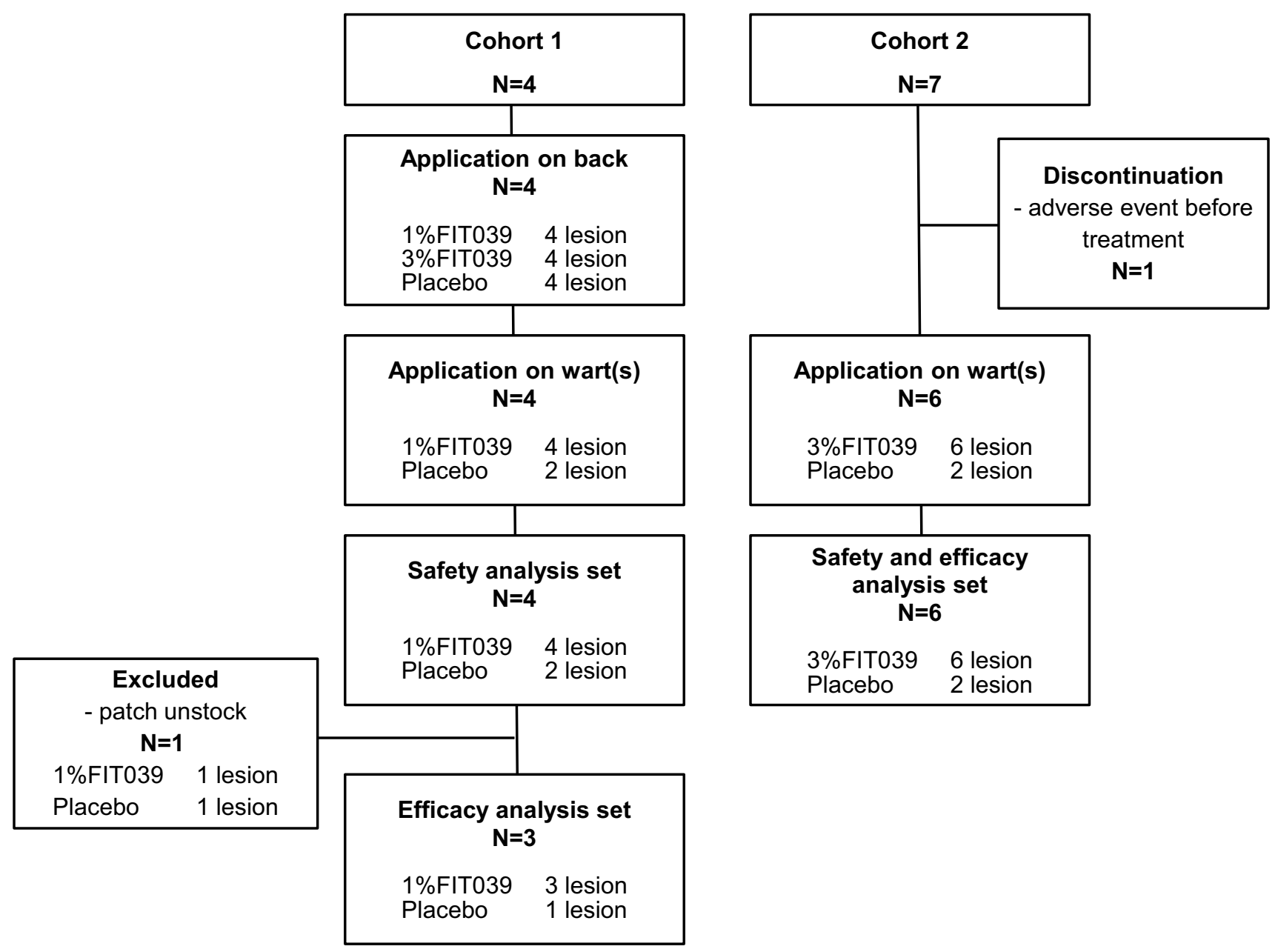

Fig. 1 Flow diagram of the patients and treated lesions for the phase I/II clinical trial of the FIT039 patch 
Table 1 Baseline characteristics of the patients

\begin{tabular}{|c|c|c|c|}
\hline Characteristics & $1 \% \operatorname{FIT039}(N=4)$ & $3 \%$ FIT039 $(N=6)$ & $\operatorname{Placebo}^{\mathrm{a}}(N=4)$ \\
\hline Median age (range) & $32.5(23-69)$ & $51.5(20-59)$ & $32.0(20-57)$ \\
\hline \multicolumn{4}{|l|}{ Gender } \\
\hline Male (\%) & $3(75.0)$ & $3(50.0)$ & $3(75.0)$ \\
\hline Female $(\%)$ & $1(25.0)$ & $3(50.0)$ & $1(25.0)$ \\
\hline \multicolumn{4}{|l|}{ Site of the target wart } \\
\hline Palms (\%) & $0(0.0)$ & $0(0.0)$ & $0(0.0)$ \\
\hline Sole of the feet $(\%)$ & $2(50.0)$ & $5(83.3)$ & $2(50.0)$ \\
\hline Upper extremities other than palms (\%) & $2(50.0)$ & $1(16.7)$ & $2(50.0)$ \\
\hline Lower extremities other than soles (\%) & $0(0.0)$ & $0(0.0)$ & $0(0.0)$ \\
\hline \multicolumn{4}{|l|}{ Number of warts } \\
\hline Multiple (\%) & $2(50.0)$ & $4(66.7)$ & $4(100.0)$ \\
\hline Single $(\%)$ & $2(50.0)$ & $2(33.3)$ & $0(0.0)$ \\
\hline Previous cryotherapy (\%) & $1(25.0)$ & $5(83.3)$ & $1(25.0)$ \\
\hline
\end{tabular}

${ }^{\mathrm{a}}$ Of those patients, two were also given a 1\% FIT039 patch and another two patients were given a 3\% FIT039 patch

\subsection{Plasma Concentrations}

The calibration curve showed good linearity over the concentration of $0.1-100 \mathrm{ng} / \mathrm{ml}$ with a correlation coefficient of 0.9959 . The accuracy values of calibration standards ranged from 85.8 to $111.0 \%$ and were considered satisfactory (Supplementary Online Resource Table 2, Fig. 1). With regard to QC samples, the accuracy values ranged from 92.5 to $114.6 \%$ (Supplementary Online Resource Table 3) and were consistent with the requirements specified in advance.

Plasma FIT039 concentrations were evaluated at $4 \mathrm{~h}$ and $24 \mathrm{~h}$ after FIT039 application to back skin, and at 2, 4, and $24 \mathrm{~h}$ after FIT039 application to cutaneous warts. The concentrations of FIT039 were below $0.1 \mathrm{ng} / \mathrm{ml}$ at every time point. Therefore, absorbed FIT039 is less likely to induce systemic adverse reactions.

\subsection{Efficacy}

The surface areas and changes in target warts are shown in Table 2. The median wart surface area at 1 week after application of the 1\% FIT039 patch was similar to baseline, while that of the 3\% FIT039 patch was smaller than baseline (i.e., median change from baseline to 1 week was $7.08 \%$ ). Furthermore, the median change in wart surface area from baseline to 1 week after application of the placebo was $-14.29 \%$ (i.e., increased by $14.29 \%$ ). Nevertheless, we could not find a specific trend in the change in wart surface area, as treated warts varied with respect to their original sizes and the numbers of warts were small.

\section{Discussion}

We evaluated the safety, plasma concentrations, and efficacy of a single application of FIT039 patches in patients with cutaneous warts and found that the FIT039 patch gave no topical or systemic adverse reactions when applied to normal skin and cutaneous warts. We did not perform any statistical tests in the trial and could not conclude that a single application of FIT039 patch was effective against cutaneous warts.

Table 2 Baseline and change in surface area $\left(\mathrm{mm}^{2}\right)$ in the target warts (efficacy analysis set)

\begin{tabular}{|c|c|c|c|}
\hline Measurement & $\begin{array}{l}1 \% \text { FIT039 (no. of } \\
\text { lesions = 3) }\end{array}$ & $3 \%$ FIT039 $($ no. of lesions $=6)$ & Placebo $($ no. of lesions $=3$ ) \\
\hline \multicolumn{4}{|l|}{ Baseline } \\
\hline Median (range) & $9.00(4-30)$ & 22.38 (4 to 270$)$ & $7.20(5$ to 35$)$ \\
\hline \multicolumn{4}{|c|}{ One week after application } \\
\hline Median (range) & $9.00(4-20)$ & 25.20 (3 to 225$)$ & $8.75(4$ to 40$)$ \\
\hline \multicolumn{4}{|c|}{ Change in surface area $(\%)$} \\
\hline Median (range) & $0.00(0.00-33.33)$ & $7.08(-25.00$ to 25.00$)$ & $-14.29(-21.53$ to 20.00$)$ \\
\hline
\end{tabular}


FIT039 suppresses replication of DNA viruses through inhibition of mRNA transcription [8], but does not kill DNA viruses. We assume that FIT039 must be applied for long durations so that infected epithelial cells can be turned over. This study was the first human study using FIT039, and is the initial stage of further development of the FIT039 patch as a first-line therapy for cutaneous warts. Single application of the FIT039 patch is considered safe enough to be applied repeatedly on warts in the next clinical trial.

Dermal application of a drug is an efficient way to increase the skin concentration of the drug without exposing the whole body to the drug. However, the permeation of the drug is affected by the thickness of the skin layers, which differs depending on race, age, and anatomical site [12, 13]. We previously reported the pharmacokinetics of both dermal application and intravenous administration of $\left[{ }^{14} \mathrm{C}\right]$-labeled FIT039. In the study report, $\mathrm{AUC}_{\text {last }}$ was $22,300 \pm 100 \mathrm{ng}$ eq. of FIT039/ml when $1.5 \mathrm{mg} /$ body of $\left[{ }^{14} \mathrm{C}\right]$ FIT039 was administered intravenously, $\mathrm{AUC}_{\text {last }}$ was $381 \pm 65 \mathrm{ng}$ eq. of FIT039/ml or $1830 \pm 270 \mathrm{ng}$ eq. of FIT039/ml when $0.5 \mathrm{mg} /$ body of $\left[{ }^{14} \mathrm{C}\right]$ FIT039 was applied on normal or decornified skin, respectively. We applied the FIT039 patch on warts after cryotherapy expecting that transdermal permeation of FIT039 would be increased by cryotherapy, since cryotherapy transiently affects the epidermis and evokes inflammation [14]. Actually, common warts are focal epidermal hyperplasia with hyperkeratosis, and cutting of epidermal hyperplasia might be necessary to increase permeation of FIT039 when used in clinical practice, especially warts on the palm of the hand or on the sole of the foot.

After topical application, a portion of FIT039 might be metabolized in the stratum corneum and epidermis. However, the contribution of metabolizing enzymes in the skin would be less than that in the liver, since the relative expression levels of metabolizing enzymes, such as cytochrome $\mathrm{P} 450$, are much lower in the skin compared to the liver. In addition, we found that at least some of FIT039 applied on decornified rat skin penetrated through the skin in a penetrating assay [9].

Multiple cutaneous warts, sometimes located close to one another, are often found in a single patient. Six out of ten patients had multiple warts in our study. Cutaneous warts are lesions within the epidermis that are caused by HPV infection via direct skin contact through small scratches in the epidermis, not via blood flow. We evaluated the safety and efficacy of the FIT039 patch compared to those of placebo in one patient with multiple warts at the same time. The FIT039 patch is only $1 \mathrm{~cm}$ square and is not considered to affect other warts more than $5 \mathrm{~cm}$ distant from the application site. We decided in advance that adverse skin reactions at application sites were attributed to the applied patch and other adverse reactions were attributed to FIT039 in patients who received both FIT039 patches and placebos.
Efficacy of treatments against cutaneous warts is usually determined when the warts are no longer visible or remain visible after consecutive treatments for several weeks. We hypothesized that the early shrinkage of target warts calculated by the product of the long axis and short axis of the wart would predict the clearance of the wart. Earlier and more precise evaluation for effectiveness is warranted, especially in the early phase of clinical development.

Cutaneous warts are often located on the fingers and soles of the feet, where patches may peel off easily without a cover. However, only one patient reported detachment of the study patch. Therefore, adherence of the study treatment can be good when the patch is firmly covered by a film.

\section{Conclusion}

The FIT039 patch produced no topical or systemic adverse reactions when applied to normal skin or cutaneous warts. The safety and good adherence of the FIT039 patch are encouraging and support further studies to evaluate the efficacy of FIT039 in patients with cutaneous warts.

Acknowledgements We are grateful to all the participants and site personnel who took part in the study.

Author Contributions ES, TN, RA, TS, MH, and KK participated in the study design. ES wrote the manuscript. TN, YA, AY, and KK participated in the management and conduct of the study. RU and HT participated in the data management and statistical analysis. All authors read and approved the final manuscript.

\section{Compliance with Ethical Standards}

Funding This was an investigator-initiated study and was financially supported by the Translational Research Network Program of the Japan Agency for Medical Research and Development (AMED) and KinoPharma Inc. (Tokyo, Japan). The funders did not contribute to data collection, analysis, this report, or the decision to publish.

Conflict of interest MH owns equity in and is a scientific advisor of KinoPharma, Inc. The other authors declare that they have no competing interests.

Ethical approval All procedures performed in studies involving human participants were in accordance with study protocol, the Ministerial Ordinance on GCP for Drugs, and with the 1964 Helsinki Declaration and its later amendments or comparable ethical standards. The study protocol (code FIT-039-001) was approved by the IRB at Kyoto University Hospital on 12 February 2016 (K025) and the Pharmaceuticals and Medical Devices Agency (PMDA).

Informed consent Informed consent was obtained from all individual participants included in the study.

Open Access This article is distributed under the terms of the Creative Commons Attribution-NonCommercial 4.0 International License (http://creativecommons.org/licenses/by-nc/4.0/), which permits any 
noncommercial use, distribution, and reproduction in any medium, provided you give appropriate credit to the original author(s) and the source, provide a link to the Creative Commons license, and indicate if changes were made.

\section{References}

1. Bruggink SC, de Koning MN, Gussekloo J, Egberts PF, Ter Schegget J, Feltkamp MC, et al. Cutaneous wart-associated HPV types: prevalence and relation with patient characteristics. J Clin Virol. 2012;55(3):250-5.

2. Hagiwara K, Uezato H, Arakaki H, Nonaka S, Nonaka K, Nonaka $\mathrm{H}$, et al. A genotype distribution of human papillomaviruses detected by polymerase chain reaction and direct sequencing analysis in a large sample of common warts in Japan. J Med Virol. 2005;77(1):107-12.

3. Kwok CS, Gibbs S, Bennett C, Holland R, Abbott R. Topical treatments for cutaneous warts. Cochrane Database Syst Rev. 2012;9:CD001781.

4. Bruggink SC, Gussekloo J, Berger MY, Zaaijer K, Assendelft WJ, de Waal MW, et al. Cryotherapy with liquid nitrogen versus topical salicylic acid application for cutaneous warts in primary care: randomized controlled trial. CMAJ. 2010;182(15):1624-30.

5. Meinhart A, Kamenski T, Hoeppner S, Baumli S, Cramer P. A structural perspective of CTD function. Genes Dev. 2005;19(12):1401-15.

6. Okamoto M, Hidaka A, Toyama M, Hosoya T, Yamamoto M, Hagiwara M, et al. Selective inhibition of HIV-1 replication by the CDK9 inhibitor FIT-039. Antiviral Res. 2015;123:1-4.
7. Tanaka T, Okuyama-Dobashi K, Murakami S, Chen W, Okamoto $\mathrm{T}$, Ueda $\mathrm{K}$, et al. Inhibitory effect of CDK9 inhibitor FIT-039 on hepatitis B virus propagation. Antiviral Res. 2016;133:156-64.

8. Yamamoto M, Onogi H, Kii I, Yoshida S, Iida K, Sakai H, et al. CDK9 inhibitor FIT-039 prevents replication of multiple DNA viruses. J Clin Invest. 2014;124(8):3479-88.

9. Ajiro M, Sakai H, Onogi H, Yamamoto M, Sumi E, Sawada T, et al. CDK9 inhibitor FIT-039 suppresses viral oncogenes E6 and E7 and has a therapeutic effect on HPV-induced neoplasia. Clin Cancer Res. 2018;24(18):4518-28.

10. The International Contact Dermatitis Research Group. http:// www.icdrg.org/. Accessed 15 Jul 2018.

11. Agency PMDA. Guideline on Bioanalytical Method Validation in Pharmaceutical Development. 2013. https://www.pmda.go.jp/files /000206209.pdf. Accessed 17 Sept 2018.

12. Oesch F, Fabian E, Guth K, Landsiedel R. Xenobiotic-metabolizing enzymes in the skin of rat, mouse, pig, guinea pig, man, and in human skin models. Arch Toxicol. 2014;88(12):2135-90.

13. van Eijl S, Zhu Z, Cupitt J, Gierula M, Gotz C, Fritsche E, et al. Elucidation of xenobiotic metabolism pathways in human skin and human skin models by proteomic profiling. PLoS One. 2012;7(7):e41721.

14. Sterling JC, Gibbs S, Haque Hussain SS, Mohd Mustapa MF, Handfield-Jones SE. British Association of Dermatologists' guidelines for the management of cutaneous warts 2014. Br J Dermatol. 2014;171(4):696-712. 